\title{
Surgical Knot Strength in Continuous Wound Closures
}

\author{
Anthony Nigliazzo, Rodrigo Arrangoiz, Richard Hutchison, Marc Basson, Andrew Saxe \\ Department of Surgery, Michigan State University College of \\ Human Medicine East Lansing, Michigan, USA \\ E-mail: andrew.saxe@chm.msu.edu
}

Received February 4 2011; revised April 25 2011; accepted May 192011

\begin{abstract}
Background: Forces applied to knots used for interrupted vs. continuous closures are very different. We studied the knot strength and knot security of three knots when simulating a continuous wound closure: the square, the sliding, and a hybrid constructed using a surgeon's square knot followed by a sliding knot. Materials and Methods: Knot holding capacity (KHC) of single-strand 1-0 polypropylene was determined by slow distraction on a horizontal testing sled of the strand that would be used to complete a continuous ("running") closure following placement of an anchoring knot with six throws. Distraction continued until failure of the knot defined as breakage or slippage of the knot. Results: The mean and standard deviation of KHC measured in pounds was determined $(n=30$ for each knot): standard square $8.94+/-1.04$; sliding $10.72+/-1.35$; and hybrid $10.95+/-1.10$. For each knot the relative knot security [(KHC of the knot/Tensile strength of untied strand) $\times 100$ ] was calculated: standard square $69.5 \%$; sliding $83.4 \%$; hybrid $85.2 \%$. Significant differences $(\mathrm{p}<0.0001)$ in KHC exist between square and sliding knots (favoring sliding knots) and between square and hybrid knots (favoring hybrid knots). Hybrid and sliding knots were not statistically different. Conclusions: Sliding knots and hybrid knots are superior to square knots as anchoring knots for single-strand continuous wound closure.
\end{abstract}

Keywords: Knot Strength, Continuous Suture, Square Knot, Slip Knot

\section{Introduction}

Traditionally, the square knot has been taught as the ideal anchoring surgical knot regardless of its use in continuous closure or in an interrupted suture. However, the mechanical demands on knots differ between interrupted closures and continuous suturing techniques. We hypothesized that a hybrid knot, an initial square knot followed by slip knots, is the best choice for a continuous suture; that the hybrid knot would combine the square knot's resistance to slip with the strength of the sliding knot. Our study is the first to compare knot security of standard knots to a novel hybrid knot using an appropriate model for simulating the forces applied to the anchoring knot of a continuous suture.

\section{Materials and Methods}

Because of its wide and successful use 1-0 polypropylene (Prolene; Ethicon, Langhorne, PA) was chosen as suture material.
Ninety sutures were hand tied by one surgeon. Thirty knots of each type were tied: square, sliding, and a hybrid. Thirty strands were left untied to test the intrinsic tensile strength of the suture. All knots were tied with six throws. The hybrid knot was fashioned by tying a surgeon's square knot followed by 4 sliding knots. (Figure 1).

Knots were tested on a horizontal sled type materials tester. A 0.5 inch loop was placed around a hook with the axial strand (that which would participate in the continuous closure) friction locked around a second hook. (Figure 2) The force applied to each knot was measured by an Accuforce Cadet $0-50 \pm 0.03 \mathrm{lb}$ force gauge (Ametek, Paoli, PA). The force gauge was calibrated immediately prior to the project and the force gauge was "zeroed" prior to each knot or strand tested. The continuous closure strand was distracted at less than 5 $\mathrm{cm} / \mathrm{min}$ until failure. Failure was defined as slippage and/or breakage of the knot. The force applied at the time of failure was termed the knot holding capacity (KHC). MiniTab (Minitab Inc., State College, PA) statistical 


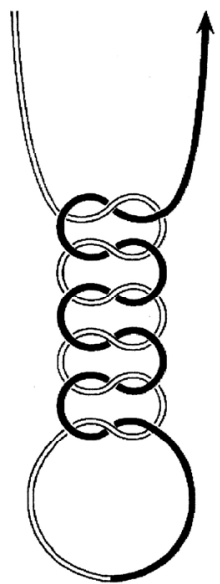

(a)

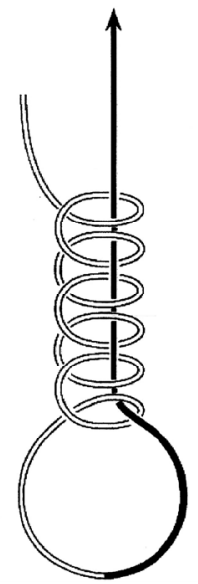

(b)

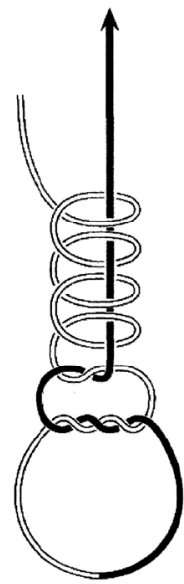

(c)
Figure 1. Illustrations of the knots tested in this study. (a) Square knot, (b) Sliding knot, (c) Hybrid knot.

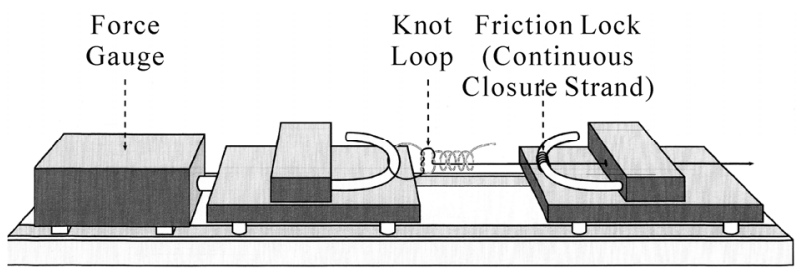

Figure 2. Illustration of the testing device.

software was used for data analysis. One-way ANOVA with post hoc pair wise analysis using a Bonferroni adjustment was performed to compare the means of KHC of the three types of knots. $\mathrm{P}$ values of $<0.05$ were considered significant.

\section{Results}

Comparisons of the knot holding capacity for each type of knot, measured in pounds, are displayed in Table 1. The hybrid knot demonstrated the greatest knot holding capacity and was statistically superior to the square knot although not statistically significantly superior to the sliding knot.

For each knot the relative knot security, defined as [(KHC of the knot/tensile strength of the untied strand) $\mathrm{X}$ 100], was calculated and is displayed in Table $\mathbf{1}$. Results were similar to those testing knot holding capacity because the tensile strength of the suture material (12.8 pounds) was the same for each type of knot.

Failure of square knots and hybrid knots occurred exclusively by breakage. Sliding knots failed by either breakage (23 of 30 knots) or slippage (7 of 30 knots).

\section{Discussion}

Several authors have investigated the properties of dif- ferent knot types when applied to tissue approximation. The majority of these studies used one of two testing methods described by Dinsmore [1]: the loop method and the single strand method. These are appropriate for interrupted sutures where the approximation of tissue is performed by a loop of suture material and the knot is stressed equally on both sides.

These methods are not appropriate, however, for the study of knots securing a continuous suture. In this setting the knot has force applied to one but not the other of the strands that exit the top of the knot. The asymmetrical force applied to the knot in the running application differs greatly from the symmetrical forces applied within the loop to the base of the knot employed in an interrupted suture. In addition to asymmetry of the forces applied to the knot of a continuous closure, there are shear forces spread along the length of the closure. These tend to elongate the suture and apply additional stress to the knot. Our study employed a model that appropriately simulated a continuous closure. We hypothesized that a hybrid knot, an initial square knot followed by slip knots, is the best choice for a continuous suture.

That our study demonstrated the superiority of sliding to square knots seems at first to be inconsistent with conventional wisdom. Sliding knots have drawn criticism on two fronts. First, sliding knots may tighten the loop. This property may lead to constriction of tissue within the loop of the knot leading to necrosis and failure of the anchor point. This could permit the running suture to loosen and ultimately lead to dehiscence of the approximated tissue. Second, sliding knots, used in the traditional interrupted fashion, may allow the knot to untie as the tissue becomes edematous increasing the diameter of the loop and pulling the exiting strands through the knot. These concerns, however, are not applicable to running closure as the suture completing the closure is the load bearing strand. It continues the length of the incision to be anchored at the opposite end and cannot come undone at the initial knot.

Others, also, have questioned the superiority generally afforded the square knot. Schaaf et al. performed a study in some ways similar to ours [2]. They calculated the relative knot security (calculated as we did) of square knots used as the starting knots, square knots used as ending knots, and Aberdeen knots. Aberdeen knots with three "throws" and one "turn" and those with four "throws" and one "turn" had superior RKS to square knots. The values they reported for square knots (approximately $60 \%-70 \%$ ) and those for the Aberdeen knots (approximately 80\%) are strikingly similar to those we found with square and hybrid knots. Richey and Roe investigated a variety of knots placed in tissue and tested in-vitro [3]. They, too, endorsed an Aberdeen knot (what 
Table 1. Properties of knots used in the setting of a continuous closure.

\begin{tabular}{cccccc}
\hline \multirow{2}{*}{ Type of knot } & $\mathrm{n}$ & \multicolumn{2}{c}{ Knot Holding Capacity (pounds) } & \multicolumn{2}{c}{ Relative Knot Security (\%) } \\
\cline { 3 - 6 } & & Mean & Standard Deviation & Mean & Standard Deviation \\
\hline Square knot & 30 & 8.9 & 1.0 & 69.8 & 8.1 \\
Sliding knot & 30 & 10.7 & 1.4 & 83.8 & 10.5 \\
Hybrid knot & 30 & 11.0 & 1.1 & 85.5 & 8.6 \\
\hline
\end{tabular}

they termed a "chain-stitch knot") although the number of throws was not provided. They found no slippage in chain-stitch knots, again similar to our findings with a hybrid knot. Aanning et al. found that a running polypropylene suture anchored with half hitches was stronger than a running suture tied with square knots [4]. Gunderson found that half-hitch knots were as secure as square knots and easier to tie [5]. Fong et al. found that abdominal wall closure with a running monofilament suture was stronger when a loop knot was used as anchor compared to a square knot or surgeons' knot [6]. Trimbos et al. tested three types of sliding knots and found that when compared to earlier data obtained using square knots there was little difference in reliability and strength between sliding knots and square knots [1]. Finally, in an extensive review Dinsmore et al. demonstrated great variation in the efficacy of different surgical knots and emphasized the difficulty in comparing studies because of lack of standardization in knot terminology and knot testing methodology [1].

There are several limitations of this study. There are innumerable variations of knots and we studied only three. We did, however, select two that are commonly used and one that we hypothesized might be superior. Also, as an ex-vivo study it did not incorporate the potential effects of in-vivo degradation and lubrication upon knot holding capacity. Finally, also as a consequence of conducting an ex-vivo study we did not account for the effect of suture placement technique.

\section{Conclusions}

Anchoring knots used for continuous abdominal wall closures experience asymmetrical forces applied to the knot. The hybrid knot best resists deformation and has the favorable knot security characteristics of both square and sliding knots. It should be used as the anchoring knot for single strand continuous abdominal wall closure.

\section{Acknowledgements}

The authors express gratitude for the graphic artwork of Mr. William Joy and the statistical analyses by Dr. Alan Davis

\section{References}

[1] R. C. Dinsmore, "Understanding Surgical Knot Security: a Proposal to Standardize the Literature," Journal of the American College Surgeons, Vol. 180, 1995, pp. 689699.

[2] O. Schaaf, M. Glyde and R. E. Day "In vitro Comparison of Secure Aberdeen and Square Knots with Plasma- and Fat-Coated Polydioxanone," Veterinary Surgery, Vol. 39, No. 5, 2010, pp. 553-560. doi:10.1111/j.1532-950X.2009.00640.x

[3] M. L. Richey and S. C. Roe, "Assessment of Knot Security in Continuous Intradermal Wound Closures,” Journal Surgical Research, Vol. 123, No. 2, 2005, pp. 284-288. doi:10.1016/j.jss.2004.08.027

[4] H. L. Aanning, T. Haas, D. R. Jorgensen and W. A. Corey, "Square not a running knot," Journal of the American College of Surgeons, Vol. 204, No. 3, 2007, pp. 422-425. doi:10.1016/j.jamcollsurg.2006.12.024

[5] P. E. Gunderson, “The Half-Hitch Knot: A Rational Alternative to the Square Knot," American Journal of Surgery, Vol. 154, 1987, pp. 538-540. doi:10.1016/0002-9610(87)90273-X

[6] E. D. Fong, A. S. R. Bartlett, S. Malak and I. A. Anderson, "Tensile Strength of Surgical Knots in Abdominal Wall Closure,” ANZ Journal of Surgery, Vol. 78, No. 3, 2008, pp. 164-166. doi:10.1111/j.1445-2197.2007.04394.X

[7] J. B. Trimbos, E. J. Van Rijssel and P. J. Klopper, "Performance of Sliding Knots in Monofilament and Multifilament Suture Material,” Obstet Gynecol, Vol. 68, 1986, pp. $425-430$. 